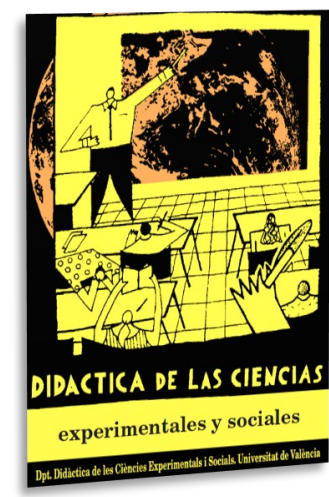

\title{
Empatía, empatía histórica y empatía prehistórica: una aproximación conceptual desde la enseñanza de las Ciencias Sociales
}

\author{
Empathy, historical empathy and prehistoric \\ empathy: a conceptual approach from Social \\ Sciences teaching
}

DOI: $10.7203 / D C E S .38 .15648$

\author{
Alberto San Martín Zapatero \\ Universidad de Burgos, jasan@ubu.es \\ ORCID iD: https://orcid.org/0000-0003-1054-1890 \\ Delfín Ortega-Sánchez \\ UniversidaddeBurgos,dosanchez@ubu.es \\ ORCID iD: https://orcid.org/0000-0002-0988-4821
}

\begin{abstract}
RESUMEN: Este trabajo presenta la evolución interdisciplinar del concepto de empatía y de sus contribuciones a la empatía histórica, con el objetivo de precisar el nuevo concepto de empatía prehistórica en el ámbito específico de la Didáctica de las Ciencias Sociales. Para ello, se revisan las aportaciones de la escuela anglosajona $\mathrm{y}$, particularmente, las desarrolladas en España desde el inicio de las corrientes de renovación de la enseñanza de la Historia hasta nuestros días, momento en que la relevancia social y educativa de la Prehistoria hace pertinente profundizar en su singularidad conceptual. La empatía prehistórica se nutre de la riqueza epistemológica del concepto de empatía, pero también dirige su mirada, desde distintos espacios educativos, a la implementación de metodologías activas, que incluyen la arqueología experimental y las técnicas propias de la dramatización.
\end{abstract}

Palabras Clave: empatía, empatía histórica, empatía prehistórica, enseñanza de las Ciencias Sociales, Prehistoria.

ABSTRACT: This study presents the interdisciplinary evolution of the concept of empathy and its contributions to historical empathy to specify the new concept of prehistoric empathy in the specific field of Social Sciences Teaching. To this end, the contributions of the Anglo-Saxon school are reviewed and, in particular, those developed in Spain since the beginning of the trends of renewal of History teaching to our days, when social and educational relevance of Prehistory makes relevant to delve into its conceptual singularity. Prehistoric empathy is nourished by the epistemological richness of the concept of empathy, but it also directs its gaze, from different educational spaces, to the implementation of active methodologies, which include experimental archeology and dramatization techniques.

KEYWORDS: empathy, historical empathy, prehistoric empathy, Social Sciences teaching, Prehistory.

Fecha de recepción: julio de 2019

Fecha de aceptación: enero de 2020

Este trabajo ha sido realizado al amparo del GIR DHISO, dirigido por el Dr. Delfín Ortega-Sánchez. 


\section{INTRODUCCIÓN}

Para definir el concepto de empatía prehistórica y singularizar el valor educativo de su significado, entendemos que se debe centrar la atención en dos factores. El primero de ellos es de carácter sociológico. Numerosos estudios demuestran el elevado interés de las sociedades occidentales por el conocimiento y valoración del pasado prehistórico (Ruiz, 2002). Este interés no es reciente; ya en la Francia revolucionaria (Pérez, 2012) o en tiempos de Darwin se constata la existencia de una estrecha relación del público con el patrimonio arqueológico (Nieto-Galán, 2011) y, hoy día, la arqueología prehistórica se adentra en la investigación de temas como la violencia, la sostenibilidad de las sociedades o la igualdad, aspectos todos ellos de interés social en el presente (Jardón y Pérez-Herrero, 2018). Los medios de comunicación, yacimientos, museos y parques arqueológicos son los canales principales de divulgación de dicho patrimonio (Ruiz, 2009).

En España, los yacimientos arqueológicos vienen ejerciendo un fuerte reclamo turístico. En los años setenta, la cueva de Altamira inaugauraba el ranking, superando en 170000 las visitas anuales, cifras superiores a las recibidas en esa década por alguno de los más importantes museos de Madrid (Fatás y Lasheras, 2014). Desde entonces, se han venido inaugurando distintos centros de interpretación y parques arqueológicos para atender la demanda de un público cada vez más numeroso. De hecho, no hay comunidad autónoma que no cuente con un centro donde se realicen talleres de simulación de carácter experimental sobre arqueología con finalidad didáctica (Gurruchaga, 2005). Uno de los primeros fue el de la Ciutadela Ibérica de Calafell en 1995 (Cardona, 2008). En conjunto, todos ellos contribuyen a difundir una imagen rigurosa o, al menos, no distorsionada del pasado prehistórico (Almansa, 2006), esto es, lo que Santacana, Llonch y Martín (2018) denominan "espacios de ocio inteligente" (p. 607).

Hoy, el fenómeno de Atapuerca representa un caso paradigmático de popularización científica de hallazgos sobre el pasado prehistórico (Hochadel, 2013). En buena medida, el éxito ha consistido en el diseño de una efectiva estrategia comunicativa que sitúa a Atapuerca en el centro de la atención pública (Nieto-Galán, 2011). En 2017, el conjunto de centros que forman el sistema Atapuerca recibió la considerable cifra de 625281 visitas (Museo de la Evolución Humana, 2017).

El segundo factor es que la Prehistoria y la Arqueología reúnen un evidente valor educativo (Bardavio y Mañé, 2017; Confortí, 2010; Ruiz, 2010; Santacana, 1999; Santacana, 2012; Santacana, Llonch y Martín, 2018). Ambas disciplinas ejercen una especial atracción sobre el público, particularmente el infantil por su capacidad para emocionar (Santacana, 2012); y ello se debe a la oportunidad que brindan para desplegar un rico abanico de actividades manipulativas (González, 2011), que tienen que ver con la didáctica del objeto, las teatralizaciones, la narración oral escénica, los juegos de rol (González, 2011), y lo que Bardavio y Mañé (2017) denominan experimentación didáctica en arqueología para referirse al método de simulación dirigido al "saber hacer" y "saber interpretar" que en el aula consiste en promover

La observación, el análisis y la reflexión sobre los restos materiales del pasado y su interpretación para reproducirlo, su representación física, la subsiguiente acción sobre la representación con una gran participación interdisciplinar y la valoración de los efectos que esta acción produce sobre los aprendizajes humanos (p.337).

Los dos factores mencionados, el sociológico y el educativo, justifican la pertinencia del concepto de empatía prehistórica, máxime cuando el término empatía ha demostrado ser extraordinariamente rico y objeto de revisión a lo largo de la historia del pensamiento, siempre en conexión con distintos ámbitos de conocimiento, entre ellos, la filosofía, la psicología, la teoría política y la didáctica de la Historia.

Este artículo pretende, por un lado, revisar la evolución terminológica, la incidencia y la aplicación del concepto de empatía al ámbito de los estudios sociales $\mathrm{y}$, en particular, en el de la 
educación histórica y, por otro, precisar el reciente concepto de empatía prehistórica desde la enseñanza de las ciencias sociales.

\section{CONCEPTO DE EMPATÍA}

Desde una vertiente estética, los orígenes de la noción de empatía pueden intuirse en el ámbito del pensamiento de tradición griega, en particular, en el Arte Poética de Aristóteles, donde la piedad y la compasión (E $\mathrm{E} \lambda \varepsilon \mathrm{s}$ ) son componentes emocionales de la tragedia, que promueven la identificación de los espectadores con los actores (López-Domínguez, 2016). También en las obras de Platón, que inaugurarán una filosofía de la persona desde las relaciones entre el yo y los otros (Ruiz de la Presa, 2007), y que tendrá notable influencia, en los albores de la Edad Media, en intelectuales como San Agustín y Santo Tomás (Revilla, 2010).

Durante los primeros momentos de la colonización europea de América, Dussel (2007) sitúa en las obras de Bartolomé de las Casas (1484-1566), Francisco de Vitoria (1483-1546) y Francisco Suárez (1548-1617) el desarrollo de un ejercicio de tener en cuenta al otro refiriéndose a las y los indígenas, víctimas de abusos y crueldades o, tal y como él mismo lo define, un respeto a la alteridad, a través de la cual se propone un encuentro armonioso desde el diálogo y el reconocimiento al otro, con el fin de frenar los abusos de los conquistadores.

Entre los siglos XVII y XVIII, Leibniz o Rousseau, para quien el ejercicio de la empatía es central en su teoría política del contrato social (López-Domínguez, 2016), sientan las bases filosóficas ilustradas del buen ciudadano, considerando la necesidad de situarse en el lugar del otro para una buena convivencia. Adam Smith, por su parte, se referiría a ella, desde un posicionamiento con resonancias de raíz protestante, como la capacidad de sentir compasión frente al dolor ajeno (Fernández-Pinto, López-Pérez y Márquez, 2008).

Asociado al campo de la estética, durante el Romanticismo alemán aparecerá el término einfühlung, entendido como la capacidad de proyectarse en el objeto artístico como contenedor de belleza (Davis, 2018). A comienzos del siglo XX, el filósofo y psicólogo alemán, Theodor Lipps, lo aplicará a la facultad de una persona de proyectarse en otra, mientras que el psicólogo británico Edward Titchener lo hará desde la voz inglesa empathy como traducción de einfühlung (Davis, 2018; Goldstein y Michaels, 1985; Rifkin, 2010; Wispé, 1987). A partir de este momento se generalizará el concepto como la tendencia a sentirse uno mismo dentro de una situación (Wispé, 1987).

Nuevas perspectivas en el estudio de la empatía se irán abriendo camino en las primeras décadas del siglo XX. Uno de sus primeros representantes del enfoque cognitivo fue Wolfgang Köhler (1929), para quien la empatía era el resultado de la comprensión de los sentimientos y no tanto el intercambio afectivo entre las personas (Demetriou, 2018). Desde este enfoque, Jean Piaget señalaba que la capacidad del niño y de la niña de descentrarse, de comprender la perspectiva de la otra persona, era una habilidad que se presentaba en un determinado momento de su desarrollo cognitivo y que la habilidad de empatizar con otros aumentaba con la edad, aspecto trascendental para su desarrollo en sociedad (Piaget, 1983). En esta línea, estudios posteriores relacionarán la empatía con el desarrollo de la competencia social (Howes, Matheson, y Hamilton, 1994; MunsonSimeo, 2000). Y otros, como Orjuela et al. (2010) y Melgarejo y Ramírez (2006) (citados en Muñoz y Chaves, 2013), sostendrán que la empatía permite reconocer los estados emocionales, lo que posibilita la interacción entre las personas.

En los albores de los años 70 del siglo XX, Hogan (1969) señalaría que la empatía se refiere a la posibilidad de acceder al modo de pensar de la otra persona, de tomar contacto con otros puntos de vista. Dentro de esta corriente cognitiva, se consolidará el concepto de adopción de perspectiva $\mathrm{o}$, en su término inglés role-taking, como la capacidad de imaginar los pensamientos y motivos de los otros (Davis, 2018). 
A finales de la década de los 60 y comienzos de los 70, comenzaba a ver la luz una nueva visión sobre la empatía centrada en el componente afectivo. Esta corriente daba mayor importancia al aspecto afectivo que al cognitivo, señalando que las personas expresan respuestas emocionales de carácter vicario frente a situaciones emocionales ajenas. Esto es, mostrar un sentimiento que otra persona experimenta (Etxeberria y Caba, 1998; Hoffman, 1987; Kohn, 1990; Mehrabian y Epstein, 1972; Stotland, 1969; Stotland, Sherman y Shaver, 1971).

Nuevos estudios en los años 80 irán construyendo una definición de empatía que conciliará el enfoque cognitivo con el afectivo (Barrio, Holgado y Carrasco, 2012; Fernández-Pinto, LópezPérez y Márquez, 2008). Desde esta perspectiva, Davis (1980) fijará un nuevo marco de interpretación conceptual al proponer una definición multidimensional de empatía. Al mismo tiempo, diseñará un instrumento metodológico, Interpersonal Reactivity Index (IRI), que buscará evualuar las dimensiones cognitiva y emocional de la empatía, útil en diversos contextos, entre ellos, el educativo. Estos intentos de desarrollar herramientas para la medida de la empatía empezarán a ser utilizados, adaptados y mejorados (Carril, Sánchez-Agustí y Miguel-Revilla, 2018; Pérez-Albéniz, De Paúl, Etxeberría, Paz y Torres, 2003; Mestre, Frías y Samper, 2004).

El estudio de la empatía se amplía, incluso, a campos como el del medio ambiente. Lo trabajos de Shelton y Rogers (1981) llamaron la atención sobre la posibilidad de proteger formas de vida no humanas a través del ejercicio de la empatía. Esta nueva dimensión de la empatía contribuirá al ámbito educativo, especialmente, a las áreas de Ciencias sociales y Ciencias de la Naturaleza en lo referente al tratamiento de valores relacionados con el cuidado, la protección y el respeto del entorno.

Por su repercusión, especialmente, en el ámbito de la psicopedagogía, la obra de Gardner, Frames of Mind (1983), merece una mención destacada. Gadner definió ocho inteligencias, entre ellas, la inteligencia interpersonal, concebida como la capacidad para comprender a otras personas (Gardner, 1995). Salovey y Mayer (1990), a partir de estos estudios, definirán la inteligencia emocional valorando la empatía como una capacidad de dicha inteligencia. La relevancia de estas investigaciones favorecerá la construcción de un concepto de empatía de carácter sincrético.

De forma sucinta, Davis (1996) entenderá la empatía como "el conjunto de constructos que incluyen los procesos de ponerse en el lugar del otro, y respuestas afectivas y no afectivas" (p.12). La empatía, como concepto, ha seguido enriqueciéndose desde finales de la década de los 1990 en el marco de los estudios sobre inteligencia emocional. Goleman (2012) identifica tres tipos de empatía: la empatía cognitiva, que permite ver la perspectiva de la otra persona; la empatía emocional como base de la compenetración; y la preocupación empática, que nos percata de que alguien necesita ayuda.

En buena medida, la neurociencia, a lo largo de los años, ha hecho un aporte sustantivo al estudio de la empatía (Decety y Jackson, 2004; Goleman 2012; Rizzolatti y Sinigaglia, 2006; Shamay-Tsoory, Tomer, Goldsher, Bergery Aharon-Peretz, 2004). Las bases neurológicas han permitido situarla en regiones diferenciadas del cerebro desde una perspectiva neuropsicológica (Fernández-Pinto, López-Pérezy Márquez, 2008). En este sentido, el hallazgo de las neuronasespejo ha sido relevante, pues ha posibilitado reconocer la existencia de mecanismos que permiten reconocer y comprender las intenciones de otras personas (Albiol, Herrero y Bernal, 2010). Esta capacidad de percibir las intenciones de los otros define al ser humano como agente intencional, lo que significa que interactuamos por el hecho de reconocer metas comunes, poniendo en marcha mecanismos de cooperación, de colaboración y de imitación, elementos clave en la conformación de nuestra historia cultural (Tomasello, 2007).

\section{EMPATÍA HISTÓRICA Y ENSEÑANZA DE LA HISTORIA}

La incorporación del concepto de empatía en la Didáctica de las Ciencias Sociales se produce, de forma paralela, a su formación como disciplina autónoma en los años ochenta. A lo largo de las 
décadas 70 y 80, se despierta en Europa una clara vocación renovadora que terminará por consolidar la Didáctica de las Ciencias sociales como una disciplina con preocupaciones investigadoras específicas (Adúriz-Bravo e Izquierdo, 2002). Los orígenes de este impulso innovador en países como Alemania o Reino Unido se encuentran en la crisis de la enseñanza de la Historia tras la Segunda Guerra Mundial (Rüsen, 2007).

En Alemania, fueron significativas las aportaciones de la teoría crítica de la Escuela de Frankfurt, que tuvieron repercusión no solo en la investigación histórica, sino también en los debates que, en los años 80, se dieron en torno a la renovación de la didáctica de la Historia (Cataño, 2011). Próximo a la Escuela de Frankfurt, nace el concepto de empatía con el vencedor de Benjamin (Benjamin y Pericay, 2007), quien, desde una visión crítica, construye este concepto a partir de la tradición positivista e historicista alemana. Desde su perspectiva, el historiador debe ser crítico y estudiar el pasado desde diferentes perspectivas, incluida la del vencido.

Las experiencias traumáticas que jalonaron el siglo XX requirieron un proceso de reflexión profunda en Alemania. Se estimuló el desarrollo de la didáctica de la Historia en relación y diálogo permanente con los estudios historiográficos y pedagógicos (Cataño, 2011). La República Federal Alemana (RFA) inició una reforma profunda en el currículum de Ciencias sociales traducida en objetivos que contemplaban la participación crítica del alumnado en el conocimiento de las estructuras de la realidad social, utilizando metodologías activas (Escolano, Jiménez y Cerezo, 1991). Sobre estas bases han destacado posteriormente las contribuciones de Rüsen (2007) en torno a las ideas de memoria histórica y conciencia histórica para el desarrollo del pensamiento histórico (Miguel-Revilla y Sánchez-Agustí, 2018).

En el Reino Unido de la década de los 70, se publicaron una serie trabajos desarrollados en los principios de la psicología evolutiva de Piaget. Estos estudios venían a expresar la necesidad de retardar en el currículum escolar la materia de Historia hasta aproximadamente los 16 años, momento en que se consideraba maduro el pensamiento socio-histórico del alumnado (Barca, 2001; Fuentes, 2002). Todavía hoy, llama la atención observar cómo persisten concepciones y prácticas entre determinado profesorado y guías arqueológicos, que consideran que los temas relativos a la Prehistoria no son aptos para su comprensión entre el público infantil (Barca, 2001).

La perspectiva evolucionista piagetiana comenzará a superarse con trabajos como el de Dickinson y Lee (1978), desarrollados en el marco del movimiento de renovación educativa New History y centrados en la necesidad de implementar contenidos procedimentales y metodológicos en la enseñanza de la Historia. También en esta línea, a comienzos de la década de los 80, Booth (citado en Ávila, Cruz y Díez, 2008) promoverá un avance en los estudios sobre teoría didáctica para la comprensión de la Historia, cuestionando los límites de edad establecidos por la psicología evolutiva de Piaget (Fuentes, 2002; Zaragoza, 1991).

Dentro de la corriente New History, uno de los proyectos que mejor recogió la percepción del alumnado sobre la Historia fue History 13-16. Shemilt (1980), como director del proyecto, fijaría el objetivo de conocer el entramado conceptual que el alumnado empleaba para entender la Historia (Fuentes, 2002). Como señala Domínguez (1991), se pretendía estudiar su grado de adquisición de nociones sobre la disciplina histórica como "causación, acción motivada, necesidad, cambio, continuidad, y metodología histórica: evidencia y empatía" (p. 55). En definitiva, se empezaban a sentar las bases científicas de la forma en que el alumnado construía su conocimiento sobre la Historia. En este contexto, la empatía histórica será uno de los conceptos estudiados para tal fin. En estos estudios pioneros, se buscaba la manera de que el alumnado adquiriera reciprocidad de posición y diferentes perspectivas respecto al pasado. Shemilt (1989) la definiría como la disposición o capacidad para comprender las acciones de los humanos en el pasado.

A partir de este momento, los países anglosajones se situaron a la cabeza de un gran número de estudios sobre empatía histórica (Almansa, 2018; Brooks, 2011; Davis, Yeager y Foster, 2001; Endacott 2010; Endacott y Brooks 2013; Lee, Dickinson y Ashby, 1995; Portal 1987). Se trataba de desarrollar el pensamiento histórico más allá de esquemas de interpretación presentistas (García y 
Jiménez, 2010; Portal, 1990; Seixas, 1993), es decir, de trazar estrategias didácticas que permitieran comprender el pasado desde la perspectiva de ese pasado. Para ello, se debían conjugar, en equilibrio, las vertientes cognitiva y emocional, esto es, lo que Sáiz (2013) denomina "la habilidad de poner en marcha, en un escenario debidamente contextualizado, una 'imaginación histórica controlada"” (p. 4). Por lo tanto, reconstruir un determinado contexto histórico implica realizar cierto tipo de ejercicios que activen la imaginación y que posibiliten la adopción de los puntos de vista de los y las protagonistas en su escenario histórico. Solo así, se puede superar un análisis centrado en los valores y puntos de vista propios.

El concepto de empatía aplicado a la didáctica de la Historia llega a España en los años 70 de la mano del proyecto Historia 13-16, inspirado en el espíritu renovador del proyecto británico History 13-16. Se empezaron a explorar metodologías para el aprendizaje histórico que superaran lo memorístico, y donde las destrezas y los procedimientos adquirieran un lugar destacado (Domínguez, 1986). El concepto de empatía, aplicado a la comprensión de la Historia, se populariza ya en los años 80. Domínguez (1986) identifica las dimensiones afectiva y cognitiva del concepto de empatía, y las emplea con el fin de analizar la relación entre este concepto y el grado de comprensión del pasado de los y las estudiantes de enseñanza media. En sintonía con esta línea de trabajo, vieron la luz otros muchos estudios que comenzaron a valorar el concepto de empatía como un elemento más, entre otros, necesario para lograr una comprensión histórica (Delval, 1989; Carretero y Jacott, 1993; Pozo,1985; Pozo y Carretero 1989).

Especialmente destacadas son las aportaciones que han ido gestándose en el seno del Grup de Recerca en Didàctica de les Ciències Socials (GREDICS) de la Universitat Autònoma de Barcelona. En sus trabajos, se evidencia que la empatía histórica requiere de la imaginación histórica y de la contextualización de los juicios sobre el pasado (Santisteban, González, y Pagés, 2010). También en el seno de este grupo se propone una conceptualización de la empatía en tres niveles: empatía histórica presentista, empatía histórica experiencial y empatía histórica, que demuestra que las acciones del pasado se producen en un contexto diferente al del presente (González, Henríquez, Pagès y Santisteban, 2009). En esta dirección son enriquecedoras las recientes aportaciones de Chaparro, Martínez, Robles y Cependosa (2019) sobre el desarrollo de la conciencia histórica del alumnado a partir de actividades realizadas con elementos de patrimonio urbano. Los resultados evidencian el surgimiento de vínculos emocionales entre la historia de la ciudad y las experiencias del alumnado participante, permitiendo establecer relaciones entre pasado y presente.

Identificadas las dificultades del alumnado para la comprensión del objeto de estudio de la Historia, de sus funciones y de los procedimientos del historiador (García y Jiménez, 2010), lo que se traduce en actitudes de desinterés y desmotivación hacia la materia (Feliu, Jiménez y Cardona, 2017; Rodríguez, Zapico y de los Hoyos González, 2016), Almansa (2018) propone un concepto de empatía histórica crítica, que permita al alumnado percibir determinadas problemáticas del pasado como propias y cercanas, identificar aspectos comunes entre pasado y presente que ayuden a humanizar el fenómeno histórico, y adoptar actitudes más comprometidas y críticas con los problemas del presente. Se trata de hacer una didáctica de la Historia capaz de mirar al pasado para hacer frente a lo que Rüsen (2007) denomina los desafíos de la globalización.

\section{EMPATÍA PREHISTÓRICA, ARQUEOLOGÍA EXPERIMENTAL Y JUEGO DRAMÁTICO}

Recientemente, encontramos trabajos que abordan el concepto de empatía aplicado a los procesos de enseñanza y aprendizaje de un período tan remoto y dilatado como la Prehistoria (Dorado, 2014; López-Castilla, Terradillos-Bernal y Alonso, 2019). López-Castilla, TerradillosBernal y Alonso (2017) emplean el concepto específico de empatía prehistórica para referirse al resultado de aplicar metodologías didácticas basadas en la experimentación didáctica en arqueología. La empatía prehistórica es definida como la habilidad para ponerse en lugar de otras 
personas, en distintos contextos históricos, mediante la reproducción de elementos culturales, lo que permite el desarrollo de una imaginación contextualizada (López-Castilla, Terradillos-Bernal y Alonso 2017). De acuerdo con este planteamiento, la experimentación didáctica en arqueología es un componente sigularizador de la empatía prehistórica, ya que "a diferencia de los tiempos históricos, cuyo estudio y comprensión posee un registro arqueológico que se ve complementado con textos escritos" (López-Castilla, Terradillos-Bernal, y Alonso, 2019, p.10), el conocimiento de la Prehistoria y de sus colectivos 'anónimos' que la vivieron cuenta con referentes provenientes de la cultura material y de la antropología cultural.

En efecto, la arqueología experimental permite conocer algunas de las características de la acción humana en la Prehistoria a través de su producción material (Alonso, Cuartero y TerradillosBernal, 2005; Alonso y Terradillos-Bernal, 2012). Esto invita a imaginar y a ponernos en el lugar, por ejemplo, del que realizó un fuego por fricción o fabricó una flecha. Desde un punto de vista didáctico, supone un buen ejercicio para tomar contacto con las fuentes primarias, pues requiere del niño y de la niña observar, tocar y analizar objetos concretos, intentar reproducir las técnicas y, a partir de ellas, adquirir la perspectiva necesaria para identificar y diferenciar los contextos en los que se produjeron determinadas acciones culturales. En suma, representa un ejercicio para poner en marcha una imaginación prehistórica controlada, favoreciendo la contextualización, el desarrollo de una empatía histórica experiencial, la imaginación y la estimulación de actitudes de respeto hacia otras formas de cultura (Alonso, Cuartero y Terradillos-Bernal, 2005). En definitiva, permite el reconocimiento y la existencia del otro (García, 2007), factor previo para que pueda desarrollarse la empatía, necesaria para una educación en la diversidad.

A través del estudio de determinadas conductas que tuvieron lugar en la Prehistoria, conocidas a través de la Arqueología (migraciones, enterramientos, arte, solidaridad y cuidados...), se puede inferir la presencia de emociones elementales, entendidas estas como aquellas que nos acompañaron durante nuestra adaptación en el proceso evolutivo (Rivera, 2015). Este aspecto es muy importante para contribuir a difundir una visión del hombre y de la mujer prehistóricos como seres emocionales, capaces de expresar miedo, alegría o tristeza. Trabajar la Prehistoria en el aula desde esta perspectiva permitiría rescatar una imagen más humana del hombre y de la mujer prehistóricos, ayudando a superar concepciones estereotipadas asociadas con la rudeza, la violencia o la insensibilidad. De acuerdo con Benjamin (2007), se haría preciso "cepillar la Prehistoria a contrapelo" a fin de no reproducir las visiones antropocentristas en la explicación de la evolución humana, presentes aún hoy en la sociedad (Querol, 2001; Scott, 2005). Este enfoque dota de valor educativo a la Prehistoria por cuanto contribuye a desarrollar actitudes de tolerancia, convivencia y solidaridad (Boj, 2001), así como de rechazo y denuncia del racismo y la xenofobia (Ruiz, 2010).

Trabajar la empatía prehistórica, incorporando la dimensión emocional, contribuiría a humanizar el fenómeno prehistórico. Para ello, se propone la utilización de estrategias didácticas relacionadas con la dramatización. En esta línea, son muchos los estudios que reconocen en el empleo de metodologías relacionadas con la dramatización una forma de obtener empatía histórica (Alonso, 2010; Cuenca, 2014; Cruz, Caballero, y Ruiz, 2013; Friera, 1995; García, 1993; Hernàndez, 2008; Navarro, 2007; Quinquer, 2004). El juego o acto dramático facilita, en efecto, la aproximación a las preconcepciones del alumnado sobre la Prehistoria; ayuda al y la participante a vencer la timidez (Alonso, 2010) y a acercarse al personaje prehistórico, a partir del uso de disfraces y atrezo; posibilita la identificación y toma de conciencia de un determinado problema o conflicto; ofrece un marco ideal para el desarrollo de la expresión corporal; y facilita la incorporación en escena de cualquier otra práctica como, por ejemplo, las simulaciones con base en la experimentación didáctica en arqueología.

La utilización de la dramatización para trabajar la empatía prehistórica requiere, en todo momento, la coordinación, supervisión y seguimiento de un profesorado o de un guía docente que reúna tres características. La primera de ellas, formación para el uso de la dramatización que, según Navarro (2007), ofrezca "habilidades pedagógicas (planificación, control del tiempo, estructuración 
de las sesiones, observación, implicación, atención al proceso personal y de grupo, dinamizar un grupo, revisión y evaluación)” (p. 169). La segunda, que conozca las características artísticas del drama. Y la tercera, que domine los suficientes conocimientos en Prehistoria y Arqueología para detectar errores y estereotipos en las preconcepciones de los y las participantes sobre el pasado humano (Ruiz, 2010; Ruiz y Álvarez-Sanchís, 1997).

Desde esta perspectiva, proponemos una definición de empatía prehistórica entendida como la capacidad de 'salirse de uno mismo', esto es, de realizar un ejercicio de desubicación consciente, de tomar distancia con el momento histórico presente, a través de las posibilidades que brinda el juego dramático y la experimentación didáctica en arqueología, para activar la imaginación y los conocimientos previos y, de este modo, representar en escena aspectos de la vida y de las actividades de otro ser humano que vivió el tiempo pasado de la Prehistoria. La empatía prehistórica es un intercambio de información entre las personas participantes en un clima de escucha y confianza. En definitiva, es ponerse cognitiva y emocionalmente en el lugar del que actúa como personaje prehistórico, siempre bajo la coordinación y el acompañamiento del guía o docente experto para que, en interacción, establezcan una relación mutua de aprendizaje.

\section{NOTAS FINALES}

En la presente revisión teórica se ha prestado atención al concepto de empatía histórica como uno de los conceptos clave en el desarrollo y adquisición de habilidades de pensamiento histórico. Por extensión, el concepto se ha singularizado para la etapa de la Prehistoria, término utilizado en los estudios más recientes. El contexto en el que se produce esta aportación didáctica viene caracterizado por el interés que despierta la Arqueología y la Prehistoria a nivel social, cultural y educativo.

El término empatía prehistórica se nutre de la riqueza epistemológica del concepto de empatía, pero también dirige su mirada, desde distintos espacios educativos, a la implementación de metodologías, especialmente interesantes en el ámbito de la enseñanza de las Ciencias sociales, como son: las simulaciones, las experimentaciones didácticas con base en la arqueología experimental, las técnicas propias de la dramatización en el aula y la didáctica del objeto. Aspectos todos ellos relevantes para desarrollar en el aula un aprendizaje basado en competencias, que relegue definitivamente prácticas y metodologías basadas exclusivamente en lo memorístico, lo expositivo y el libro de texto como recurso central. De forma global, estos factores posibilitan una aproximación a la Prehistoria mucho más cercana, participativa y motivadora de visiones no antropocéntricas, que invita a reflexionar sobre cuestiones socialmente relevantes y actuales de la humanidad desde los principios del aprendizaje significativo.

\section{Referencias}

Adúriz-Bravo, A. e Izquierdo, M. (2002). Acerca de la didáctica de las ciencias como disciplina autónoma. Revista Electrónica de Enseñanza de las Ciencias, 1(3), 130-140.

Albiol, L. M., Herrero, N. y Bernal, M. (2010). Bases neuronales de la empatía. Neurología de la conducta, 2(50), 89-100.

Almansa, J. (2006). La imagen popular de la arqueología en Madrid. ArqueoWeb, 8. Recuperado de https://webs.ucm.es/info/arqueoweb/ [15 de abril de 2019].

Almansa, R. M. (2018). La empatía como método humanístico de docencia de la Historia: sugerencias didácticas en un panorama de desvalorización de los estudios históricos. Enseñanza de las ciencias sociales: revista de investigación, 17, 87-98. 
Alonso, R. y Terradillos-Bernal, M. (2012). La arqueología experimental. Una nueva disciplina para el análisis del instrumental lítico. En D. Hernández y J. P. López (Eds.), Nuevos paradigmas en la investigación arqueológica (pp. 91-101). Madrid: La Ergastula Ediciones.

Alonso, R., Cuartero, F. y Terradillos-Bernal, M. (2005). II Jornadas de Arqueología Experimental. Revista Atlántica-Mediterránea de Prehistoria y Arqueología Social, 7, 251-256.

Alonso, S. (2010). Didáctica de las Ciencias sociales para la Educación Primaria. Madrid: Pirámide.

Ávila, R., Cruz, A. y Díez, C. (2008). Didáctica de las Ciencias sociales, Currículo escolar y Formación del profesorado. Jaén: AUPDCS.

Barca, I. (2001). Educaçao Histórica: uma nova área de investigaçao. Revista da Facultade de Letras. História, 2, 13-21.

Bardavio, A. y Mañé, S. (2017). La Arqueología en la enseñanza obligatoria. El ejemplo del Campo de Aprendizaje de La Noguera. Revista Otarq: Otras arqueologías, 2, 331-345.

Barrio, V., Holgado, F. P. y Carrasco, M. Á. (2012). Dimensionalidad de la empatía y su relación con depresión, agresión y prosocialidad. Revista de Psicología y Educación, 7(2), 49-67.

Benjamin, W. (2007). Tesis sobre la filosofía de la historia. València: Quaderns republicans de l'ACR Constanti Llombart.

Boj, I. (2001). La didáctica de la Prehistoria como instrumento de transformación social. Íber: Didáctica de las Ciencias sociales, Geografía e Historia, 29, 19-26.

Cardona, F. X. (2008). La ciudadela ibérica de Calafell: balance. Íber: Didáctica de las Ciencias sociales, Geografía e Historia, 57, 17-24.

Carretero, M. y Borrelli, M. (2008). Memorias recientes y pasados en conflicto: ¿cómo enseñar historia reciente en la escuela? Cultura y Educación, 20(2), 201-215.

Carretero, M. y Jacott, L. (1993). Historia y relato. La comprensión de los agentes históricos en el 'descubrimiento' de (encuentro con) 'América'. Substratum, 2, 21-35.

Carril, T., Sánchez-Agustí, M. y Miguel-Revilla, D. (2018). Perspectiva histórica y empatía: su interrelación en futuros profesores de Educación Primaria. Aula Abierta, 47(2), 221-228. doi: 10.17811/rifie.47.2.2018.221-228

Castillo, A. S. (2015). El mapa de fosas del País Vasco. Un recurso didáctico de la arqueología del conflicto basado en las TIC. Revista de Didácticas Específicas, 12, 117-135.

Cataño, C. L. (2011). Jörn Rüsen y la conciencia histórica. Historia y sociedad, 21, 221-243.

Chaparro, A., Martínez, C., Robles, F. J., y Cepedonsa, R. (2019). Desarrollar la conciencia histórica a través del patrimonio en el Grado en Educación Primaria. Didáctica de las ciencias experimentales y sociales, 36, 17-32. DOI: 10.7203/DCES.36.12672.

Confortí, M. E. (2010). Educación no formal y patrimonio arqueológico. Su articulación y conceptualización. Intersecciones en Antropología, 11, 103-114.

Cruz, V., Caballero, P. y Ruiz, G. (2013). La dramatización como recurso didáctico para el desarrollo emocional. Un estudio en la etapa de educación primaria. RIE, 31(2), 393-410.

Cuartero, F. y Alonso, R. (2005). El parque arqueológico de Atapuerca: discurso y recursos de un centro de interpretación. En Ch. de Francia y R. Erice (Eds.), De la excavación al público: procesos de decisión y creación de nuevos recursos (pp. 217-220). Zaragoza: Institución Fernando el Católico.

Cuenca, J. M. (2014). El papel del patrimonio en los centros educativos: hacia la socialización patrimonial. Tejuelo. Didáctica de la Lengua y la Literatura. Educación, 19, 76-96.

Davis, M. H. (2018). Empathy a Social Psychological Approach. New York: Routledge.

Davis, M. H. (1996). Empathy. A Social Psychological Approach. New York: Routledge.

Decety, J. y Jackson, P. L. (2004). The Functional Architecture of Human Empathy. Behavior Cognitive Neurosciences Review, 3(2), 71-100.

Delval, J. (1989). La representación infantil del mundo social. En I. Turiel, I. Enesco y J. Linaza (Eds.), El mundo social en la mente infantil (pp. 245-328). Madrid: Alianza. 
Demetriou, H. (2018). Empathy, Emotion and Education. London: Palgrave Macmillan.

Dickinson, A. K. y Lee, P. J. (1978). Understanding and Research. En A. K. Dickinson y P. J. Lee (Eds.), History Teaching and Historical Understanding (pp. 95-120). Londres: Heinemann.

Domínguez, J. (1986). Enseñar a comprender el pasado histórico: conceptos y 'empatía'. Infancia y aprendizaje, 9(34), 1-21.

Domínguez, J. (1991). El lugar de la Historia en el currículum 11-16. Un marco general de referencia. En M. Carretero, J. I. Pozo y M. Asensio (Eds.), La enseñanza de las Ciencias sociales (pp. 33-60). Madrid: Visor.

Dorado, A. (2014). La experimentación arqueológica como herramienta vehicular orientada a la mejor comprensión de la Prehistoria y la Arqueología. Arqueología y Territorio,11, 171180.

Dussel, E. (2007). Materiales para una política de la liberación. México: Plaza y Valdés.

Escolano, A., Jiménez, A. y Cerezo, J. (1991). Las Ciencias sociales en el curriculum. Perspectiva comparativista europea: los casos de Francia, Italia, Alemania y Reino Unido. En Espacio y sociedad en el ámbito autonómico (pp. 353-374). Valladolid: Junta de Castilla y León.

Etxeberria, I. y Caba, M. A. (1998). Consistencia entre cognición y acción moral: conducta solidaria en adolescentes en el contexto escolar. Infancia y Aprendizaje, 81, 83-103.

Fatás, P. y Lasheras, J. A. (2014). La cueva de Altamira y su museo. Cuadernos de Arte Rupestre, 7, 25-35.

Feliu, M., Jiménez, L.y Cardona, G. (2017). La formación competencial en Ciencias sociales para su aplicación en el aula de infantil y primaria. En R. Martínez, R. García y C. R. García (Eds.), Investigación en Didáctica de las Ciencias sociales. Retos, preguntas y líneas de investigación (pp. 125-132). Córdoba:Universidad de Córdoba-AUPDCS.

Fernández, V. M. (2012). Arqueología y patrimonio en un mundo postoccidental: estudio de dos casos de Etiopía. En C. Ferrer y J. Vices-Ferrándiz (Eds.), Construcciones y usos del pasado: patrimonio arqueológico, territorio y museo (pp. 7-30). Valencia:Museu de Prehistòria de València-Diputación de Valencia.

Fernández-Pinto, I., López-Pérez, B., \& Márquez, M. (2008). Empatía: medidas, teorías y aplicaciones en revisión. Anales de Psicología, 284-298.

Friera, F. (1995). Didáctica de las ciencias sociales. Madrid: Ediciones de la Torre.

Frutos, J. R. (Ed.) (1989). Enseñar Historia. Nuevas propuestas.. Barcelona: Laia.

Fuentes, C. (2002). La visión de la historia por los adolescentes: revisión del estado de la cuestión en Estados Unidos y el Reino Unido. Enseñanza de las ciencias sociales: revista de investigación, 1, 55-68.

García, A. L. (1993). Materiales y recursos didácticos. En A. L. Ruiz (Ed.), Didáctica de las ciencias sociales en la Educación Primaria. (pp. 97-124). Madrid: Algaida.

García, A. L. y Jiménez, J. A. (2010). El valor formativo y la enseñanza de la Historia. Granada: EUG.

García, N. (2007). Arqueología y educación. Estado de la cuestión. Cuicuilco, 14, 203-226.

Gardner, H. (1995). Inteligencias Múltiples. La teoría en la práctica. Barcelona: Paidós.

Goldstein, A. y Michaels, G. (1985). Empathy: Development, training, and consequences. New Jersey: Erlbaum Associates.

Goleman, D. (2012). El cerebro y la inteligencia emocional: nuevos descubrimientos. Barcelona: Grupo Z.

González, N., Henríquez, R., Pagès, J. y Santisteban, A. (2009). El aprendizaje de la empatía histórica en educación secundaria. Análisis y proyecciones de una investigación sobre la enseñanza y el aprendizaje del conflicto y la convivencia en la Edad Media. En R. M. Ávila, B. Borghi e I. Mattozzi (Eds.). La educación de la ciudadanía europea y la formación del profesorado. Un proyecto educativo para la estrategia de Lisboa (pp. 283-291). Bologna: Pàtron Editore. 
González, P. (2011). La dimensión educativa de la arqueología. En La tutela del patrimonio prehistórico (pp. 497-506). Málaga: Junta de Andalucía-Consejería de Cultura.

Gurruchaga, J. L. (2005). Arqueologóa, Prehistoria e historia antigua en el mundo escolar: de Isabel II al siglo XXI. Archaia, 3, 283-288.

Hernàndez, F. X. (2008). Didáctica de las ciencias sociales, geografía e historia. Barcelona: Graó.

Hochadel, O. (2013). El mito de Atapuerca. Orígenes, ciencia, divulgación. Cerdanyola del Vallès, Barcelona: El espejo y la lámpara.

Hoffman, M. L. (1987). The contribution of empathy to justice and moral judgment. En N. Eisenberg y J. Strayer (Eds.), Empathy and its development (pp. 47-80). New York: Cambridge University Press.

Hogan, R. (1969). Development of an empathy scale. Journal of Consulting and Clinical Psychology, 33, 307-316.

Howes, C., Matheson, C., y Hamilton, C. (1994). Maternal, teacher, and child care history correlates of children's relationships with peers. Child development, 65, 264-273.

Jardón, P. y Pérez-Herrero, C. I. (2018). Una Prehistoria compleja: revisión crítica de los tópicos en la representación cinematográfica para transformar su enseñanza. En E. L. Torres, C. R. Ruiz, y M. Sánchez (Eds.), Buscando formas de enseñar: investigar para innovar en didáctica de las Ciencias Sociales. (pp. 827-838). Valladolid: Universidad de ValladolidAsociación Universitaria de Profesorado de Didáctica de las Ciencias Sociales.

Köhler, W. (1929). Gestalt psychology. New York: Liveright.

Kohn, A. (1990). The brighter side of human nature: Altruism and empathy in everyday life. Nueva York: Basic Books.

Lipps, T. (1905). Das Wissen von fremden Ichen. Psychologische Untersuchungen, 4, 694-722.

López-Castilla, M. P., Terradillos-Bernal, M. y Alonso, R. A. (2017). The role of experimental archaeology in the scientific spreading as developer of prehistorical empathy. En R. Alonso, J. Baena y D. Canales (Eds.), Playing with the time: experimental archaeology and study of the past (pp. 355-360). Madrid: Servicio de Publicaciones de la Universidad Autónoma de Madrid.

López-Castilla, M. P., Terradillos-Bernal, M. y Alonso, R. A. (2019). Experimental archaeology and historical empathy: key tools for learning about our origins. Cultura y Educación, 31(1), 170-187.

López-Domínguez, V. (2016). Empatía y solidaridad transnacional. Reflexiones en torno a una conferencia de Carol C. Gould. Dilemata, 22, 283-297.

Mehrabian, A. y Epstein, N. (1972). A measure of Emotional Empathy. Journal of Personality, 40, 525-543.

Mestre, V., M.D.Frías y Samper, P. (2004). La medida de la empatía: análisis del Interpersonal Reactivity Index. Psicothema, 16, 255-260.

Miguel-Revilla, D. y Sánchez-Agustí, M. (2018). Conciencia histórica y memoria colectiva: marcos de análisis para la educación histórica. Revista de Estudios Sociales, 65, 113-125. DOI: $10.7440 /$ res65.2018.10

Munson-Simeo, J. (2000). Differences among peer status groups on levels og loneliness, social competence and empathy. (Thesis Ph.D.). University of Denver, Denver.

Muñoz, A. P. y Chaves, L. (2013). La empatía: ¿un concepto unívoco? Katharsis, 16, 123-143.

Museo de la Evolución Humana (2017). Recuperado de http://www.museoevolucionhumana.com/es/noticias-meh/el-sistema-atapuerca-incrementasu-numero-de-visitas-por-quinto-ano-consecutivo-sumando-625281-en-2017 $\quad[12 \quad$ de noviembre de 2018]

Navarro, M. R. (2007). Drama, creatividad y aprendizaje vivencial: algunas aportaciones del drama a la educación emocional. Cuestiones Pedagógicas, 18, 161-172. 
Nieto-Galán, A. (2011). Los públicos de la ciencia. Expertos y profanos a través de la historia. Madrid: Marcial Pons.

Pérez, A. (2012). El museo fuera del museo: la gestión del patrimonio arqueológico in situ. En C. Ferrer y J. Vices-Ferrándiz (Eds.), Construcciones y usos del pasado: patrimonio arqueológico, territorio y museo (pp. 115-136). Valencia: Museu de Prehistòria de València - Diputación de Valencia.

Pérez-Albéniz, A., Paúl, J. D., Etxeberría, J., Paz, M. y Torres, E. (2003). Adaptación de Interpersonal Reactivity Index (IRI) al español. Psicothema, 15, 267-272.

Piaget, J. (1983). El criterio moral en el niño. Barcelona: Fontanella.

Portal, C. (1990). Empathy. Teaching History, 5, 36-38.

Pozo, J. (1985). El niño y la historia. Madrid: Servicio de publicaciones del MEC.

Pozo, J. I., Asensio, M. y Carretero, M. (1989). Modelos de aprendizaje-enseñanza de la Historia. En M. Carretero, J. I. Pozo y M. Asensio (Eds.), La enseñanza de las Ciencias sociales (pp. 211-240). Madrid: Visor.

Pozo, J. L. y Carretero, M. (1989). Las explicaciones causales de expertos y novatos en Historia. En M. Carretero, J. A. Pozo y M. Asensio (Eds.), La enseñanza de las Ciencias sociales (pp. 139-163). Madrid: Visor.

Presa, J. R. (2007). Alteridad. Un recorrido filosófico. México: Instituto Tecnológico y de Estudios Superiores de Occidente (ITESO)-Universidad de Guanajuato-Uniersidad IberoamericanaUniversidad Iberoamericana Puebla.

Querol, M. A. (2001). De maravillosos hombres y pobres monos. Análisis del fenómeno antropocentrista en la bibliografía española sobre orígenes humanos. Cumplum, 12, 237248.

Quinquer, D. (2004). Estrategias metodológicas para enseñar y aprender ciencias sociales: interacción, cooperación y participación. Íber: Didáctica de las Ciencias sociales, Geografía e Historia, 40, 7-22.

Revilla, C. (2010). Sobre Fenomenología y Hermeneútica. Aproximación a los conceptos de empatía y comprensión. Convivium, 23, 131-152 .

Rifkin, J. (2010). La Civilización empática: la carrera hacia una conciencia global en un mundo en crisis. Barcelona: Paidós.

Rivera, A. (2015). Arqueología de las emociones. Vínculos de Historia, 4, 41-61.

Rizzolatti, G. y Sinigaglia, C. (2006). Las neuronas espejo. Barcelona: Paidós.

Rodríguez, L. B., Zapico, M. B. y González, C. D. (2016). Sentarse, escuchar y repetir. ¿Existe otra forma de enseñar historia? Ikastorratza, e-Revista de didáctica, 16, 1-26.

Armañanzas, G. (2012). Elaboración Transgeneracional del Trauma: Guerra Civil Española. Norte de Salud mental, 43, 13-17.

Ruiz-Zapatero, G. ( 2009). La divulgación arqueológica: las ideologías ocultas. Cuadernos de Prehistoria y Arqueología de la Universidad de Granada, 19, 11-36.

Ruiz-Zapatero, G. (2002). Arqueología e identidad: la construcción de referentes de prestigio en la sociedad contemporánea. ArqueoWeb, 14. Recuperado de https://webs.ucm.es/info/arqueoweb/ [6 de marzo de 2019].

Ruiz-Zapatero, G. (2010). Los valores educativos de la Prehistoria en la Enseñanza Obligatoria. Marq, arqueología y museos, 4, 161-179.

Ruiz-Zapatero, G., y Álvarez-Sanchís, J. R. (1997). La Prehistoria enseñada y los manuales escolares españoles. Complutum, 8, 265-284.

Rüsen, J. (2007). How to make sense of the past - salient issues of Metahistory. The Journal for Transdisciplinary Research in Southern Africa, 3(1), 169-221.

Sáiz, J. (2013). Empatía histórica, historia social e identidades: pensar históricamente la conquista cristiana de la Valencia musulmana con estudiantes de $2^{\circ}$ ESO. Clío, 39, 1-20. 
Salovey, P. y Mayer, J. (1990). Emotional Intelligence. Imagination, cognition, and personality, 9, $185-211$.

Santacana, J. (1999). L'arqaeologia com a eina didàctica: problemes i utilitat. Cota Zero, 15, 63-73.

Santacana, J. (2012). Arqueología, museología y comunicación. En C. Ferrer y J. Vices-Ferrándiz (Eds.), Construcciones y usos del pasado: patrimonio arqueológico, territorio y museo (pp. 137-151). Valencia: Museu de Prehistòria de València-Diputación de Valencia.

Santacana, J., Llonch, N. y Martín, C. (2018). Aprender ciencias a través de la arqueología prehistórica: una experiencia didáctica con kits educativos en el museo. ETD-Educação Temática Digital, 2018, 20(3), 604-622.

Santisteban, A., González, N.y Pagés, J. (2010). Una investigación sobre la formación del pensamiento histórico. En R. Á. Ruiz, P. R. Garcíay P. L. Domínguez (Eds.), Metodología en investigación en didáctica de las ciencias sociales. (pp. 115-128). Zaragoza: Instituto Fernando el Católico.

Scott, M. (2005). Writing the history of humanity. The role of museums in defining origins and ancestors in a transnational world. Curator, 48, 74-89.

Seixas, P. (1993). Historical Understanding Among Adolescents in a Multicultural Setting. Curriculum Inquiry, 23, 301-327.

Shamay-Tsoory, S., Tomer, R., Goldsher, D., Berger, B. D. y Aharon-Peretz, J. (2004). Impairment in Cognitive and Affective Empathy in Patients with Brain Lesions: Anatomical and Cognitive Correlates. Journal of Clinical and Experimental Neuropsychology, 26(8), 11131127.

Shelton, M. y Rogers, R. (1981). Fear-arousing and empathy-arousing appeals to help: the pathos of persuasion . Journal of Applied Psychology, 11(4), 366-378.

Shemilt, D. (1980). History 13-16. Evaluation Study. Edimburgh: Schools Council History 13-16Mc Dougall.

Shemilt, D. (1989). Beauty and the Phllosopher Emphathy in History and Classroom. En A. K. Dickinson, P. J. Lee e I. J. Rogers (Eds.), Learning History (pp. 39-84). Londres: Heinemann Education Books.

Stotland, E. (1969). Advances in experimental social psychology. En L. Berkowitz (Ed.), Exploratory investigations of empathy (pp. 271-314). New York: Academic Press.

Stotland, E., Sherman, S. y Shaver, K. (1971). Empathy and birth order: Some experimental explorations. Lincoln, NB: University of Nebraska Press.

Toledo, J., Díez, J. C. y Nastri, J. (2011). Como sobrevivir con dos piedras y un cerebro. Burgos: Servicio de publicaciones Diario de los Yacimientos de la Sierra de Atapuerca.

Tomasello, M. (2007). Los orígenes culturales de la cognición humana. Buenos Aires: Amorrortu.

Wispé, L. (1987). History of the concept of empathy. En N. Eisenberg y J. Strayer (Eds.), Empathy and Its Development (pp. 17-36). Cambridge: Cambridge University Press.

Zaragoza, G. (1991). La investigación y la formación del pensamiento histórico del adolescente. En M. Carretero, J. I. Pozo y M. Asensio (Eds.), La enseñanza de las Ciencias sociales (pp. 165-177). Madrid: Visor.

CÓMO CITAR ESTE ARTÍCULO

San Martín-Zapatero, A. y Ortega-Sánchez, D. (2020). Empatía, empatía histórica y empatía prehistórica: una aproximación conceptual desde la enseñanza de las Ciencias Sociales. Didáctica de las ciencias experimentales y sociales, 38, 3-16. DOI: 10.7203/DCES.38.15648. 
